\title{
The pattern of overnight changes in novel markers of acute kidney injury in patients with obstructive sleep apnea
}

\author{
Michał Nowicki ${ }^{1, A-F}$, Anna Zawiasa-Bryszewska, ${ }^{1, A-D}$, Małgorzata Taczykowska ${ }^{1, B}$, Piotr Białasiewicz 2,A-C,E, Dariusz Nowak ${ }^{2, A, C, E, F}$ \\ ${ }^{1}$ Department of Nephrology, Hypertension and Kidney Transplantation, Medical University of Lodz, Poland \\ ${ }^{2}$ Chair of Physiology, Medical University of Lodz, Poland \\ A - research concept and design; $B$ - collection and/or assembly of data; $C$ - data analysis and interpretation; \\ $D$ - writing the article; $E$ - critical revision of the article; $F$ - final approval of the article
}

Address for correspondence

Michał Nowicki

E-mail: nefro@wp.pl

\section{Funding sources}

The study was funded with a research grant No. N N402 559340 from the National Science Centre in Poland.

Conflict of interest

None declared

\section{Acknowledgements}

The study has been registered in the National Library of Medicine (NLM) ClinicalTrials.gov database (identifier NCT00947479).

Received on May 17, 2019

Reviewed on August 24, 2019

Accepted on June 2, 2020

Published online on September 7, 2020

Cite as

Nowicki M, Zawiasa-Bryszewska A, Taczykowska M, Białasiewicz P, Nowak D. The pattern of overnight changes in novel markers of acute kidney injury in patients with obstructive sleep apnea. Adv Clin Exp Med. 2020;29(9): 1065-1072. doi:10.17219/acem/123356

DOI

10.17219/acem/123356

Copyright

Copyright by Author(s)

This is an article distributed under the terms of the

Creative Commons Attribution 3.0 Unported (CC BY 3.0)

(https://creativecommons.org/licenses/by/3.0/)

\begin{abstract}
Background. Obstructive sleep apnea (OSA) may predispose patients to acute kidney injury (AKI) and chronic kidney disease (CKD).

Objectives. We postulated that apneic episodes during sleep in patients with OSA may result in episodes of subclinical AKI that may be detected by the use of novel sensitive serum and urine markers. The treatment of OSA may protect against renal injury.
\end{abstract}

Material and methods. The study involved 86 men who had positive screening test results for OSA and estimated glomerular filtration rates (eGFR) calculated with the Chronic Kidney Disease Epidemiology Collaboration (CDK-EPI) equation $>60 \mathrm{~mL} / \mathrm{min} / 1.73 \mathrm{~m}^{2}$. All the patients underwent overnight polysomnography (the diagnostic night). The patients were divided into 2 groups: 45 patients with mild OSA and 41 patients with moderate-to-severe OSA. Those in the latter group were qualified for $6-8$ weeks of continuous positive airway pressure (CPAP) treatment. Blood pressure, serum creatinine, high sensitivity C-reactive protein (hsCRP), urine creatinine, AKI markers cystatin ( and neutrophil gelatinase-associated lipocalin (NGAL), kidney injury molecule 1 (KIM-1), liver-type fatty acid-binding protein, and endothelium marker intercellular adhesion molecule-1 (ICAM-1) were assessed in both groups before and after polysomnography. The same parameters were also measured in the patients with moderate-to-severe OSA after CPAP therapy.

Results. Kidney injury molecule 1 and urine NGAL significantly increased after the diagnostic night in the whole group and in mild OSA patients ( $p=0.04$ and $p=0.001$, respectively). Serum ICAM- 1 significantly decreased after the diagnostic night only in mild OSA patients $(p=0.03$. Urine cystatin C increased after 6-8 weeks of CPAP treatment in the moderate-to-severe OSA group (0.003). Serum ICAM-1 decreased after the CPAP treatment $(p=0.02)$. The CPAP therapy led also to a decrease in mean and diastolic $(p=0.005)$ blood pressure.

Conclusions. The pattern of overnight changes in serum and urine AKI markers after apneic episodes during sleep may suggest an increased risk of subclinical AKI in patients with OSA. The CPAP therapy is not protective against AKl, but may reduce some of its risk factors, including high blood pressure and endothelial damage. Key words: biomarkers, acute kidney injury, obstructive sleep apnea, NGAL 


\section{Introduction}

Obstructive sleep apnea (OSA) is a common and growing clinical problem with many important negative health implications, characterized by recurrent episodes of hypoxia and hypercapnia during sleep, resulting in daytime sleepiness. ${ }^{1}$ Hypoxia and hypercapnia induced by OSA may have negative effects resulting from the activation of the sympathetic nervous system, the renin-angiotensin-aldosterone system (RAAS), and increased oxidative stress and free-radical generation. ${ }^{1-3}$ The detrimental consequences of hypoxia, which include endothelial dysfunction, systemic inflammation, platelet aggregation, atherosclerosis, and fibrosis, may predispose individuals to cardiovascular events and are likely to induce renal damage. ${ }^{2,3}$ As much as half of all patients with chronic kidney (CKD) disease suffer from nocturnal hypoxia, which has been recognized as a potential cause of chronic renal dysfunction and cardiovascular risk. ${ }^{3}$ Obstructive sleep apnea has also recently been identified as a strong risk factor for acute kidney injury (AKI) in a large population of critically ill patients. ${ }^{4}$

It has recently been suggested that frequent apneic episodes during sleep may result in repeated renal hypoxia and release of inflammatory mediators, which may cause AKI and eventually contribute to CKD. ${ }^{2,3}$ Obstructive sleep apnea has also been associated with glomerular hyperfiltration and the development of focal glomerulosclerosis, and may be an independent predictor of proteinuria, a major risk factor for CKD progression. ${ }^{5,6}$ Obstructive sleep apnea may mediate renal damage through several mechanisms, i.e., hypoxemia-induced endothelial dysfunction, accelerated atherosclerosis or altered cardiovascular hemodynamics. ${ }^{1-3}$ Therefore, there is a need to better elucidate the possible impact of OSA on the incidence of kidney injury and CKD progression.

The current routine diagnostics of AKI are based mainly on changes in serum creatinine, but these measurements may lack the sensitivity necessary to detect early and acute deterioration in kidney function. ${ }^{7}$ Serum creatinine concentration may not change until about $50 \%$ of kidney function has already been lost. Furthermore, serum creatinine levels vary widely with age, sex, muscle mass, muscle metabolism, medications, and hydration status. ${ }^{8}$ Novel, more specific and sensitive AKI biomarkers are neutrophil gelatinase-associated lipocalin (NGAL), cystatin C, kidney injury molecule 1 (KIM-1), and liver-type fatty acidbinding protein (L-FABP), the concentrations of which in urine or serum rise significantly in patients with AKI and correlate well with the severity of kidney injury.,8

The treatment of OSA with continuous positive airway pressure (CPAP) offers a unique opportunity to alleviate most maladaptive changes in the cardiovascular system. Several clinical and experimental studies have indicated that CPAP may lead to an improvement in endothelial function, decrease free radical release from neutrophils, decrease inflammatory mediators, increase vasodilator serum levels, and induce a decrease in vasoconstrictor levels in patients with sleep apnea., ${ }^{9,10}$ It is likely that CPAP treatment could mitigate renal injury and improve renal outcomes. $^{11}$

The aim of this study was to assess the effect of sleep apnea episodes on the pattern of changes in novel serum and urine markers of AKI and endothelial function in patients with different levels of severity of OSA, and to examine whether CPAP treatment of moderate-to-severe OSA could influence the overnight changes in AKI markers.

\section{Material and methods}

Ninety-nine men aged 40-67 years and having estimated glomerular filtration rate calculated with the Chronic Kidney Disease Epidemiology Collaboration (CDK-EPI) equation $\left(\right.$ eGFR $\left.\mathrm{CKD}_{\text {-EPI }}\right)>60 \mathrm{~mL} / \mathrm{min} / 1.73 \mathrm{~m}^{2}$ were referred to the Sleep and Respiratory Disorders Center in Łódź (Poland) after screening for OSA based on the Berlin Questionnaire and Epworth Sleepiness Scale (ESS) for daytime sleepiness assessment. All the patients underwent a diagnostic night: standard nocturnal polysomnography $(8 \mathrm{~h}$, from 10:00 pm to 06:00 am) using Sleep Lab (Jaeger Viasys Healthcare, Höchberg, Germany), operated by a single technician with long-term experience in polysomnography. All the readings were analyzed and interpreted by a clinical physiologist (P.B.) who was blinded to the endpoints and design of the study.

Sleep and baseline respiratory parameters were defined and scored according to the 2007 American Academy of Sleep Medicine (AASM) criteria. ${ }^{12}$ Obstructive sleep apnea was defined as $\geq 90 \%$ cessation of airflow persisting for at least $10 \mathrm{~s}$ relative to basal amplitude. Hypopnea was defined as less than or equal to $70 \%$ reduction for at least $10 \mathrm{~s}$. The apnea-hypopnea index (AHI) was calculated as the number of OSA events plus the number of obstructive hypopnea events per $1 \mathrm{~h}$ of sleep time recorded by the device. The severity of OSA was determined as mild (AHI 5-14/h), moderate (AHI 15-29/h) or severe (AHI $\geq 30 / h)$.

After positive screening tests for OSA, 86 males with apnea-hypopnea indexes $\geq 15$ episodes/h and eGFR $\mathrm{R}_{\mathrm{CKD}-\mathrm{EPI}}$ $>60 \mathrm{~mL} / \mathrm{min} / 1.73 \mathrm{~m}^{2}$ were qualified for the study. The demographic and clinical characteristics of the study population are presented in Table 1 . The exclusion criteria included proteinuria $>1.0 \mathrm{~g} / 24 \mathrm{~h} / 1.73 \mathrm{~m}^{2}$ body surface; acute and chronic inflammatory conditions including urinary tract infection (UTI), defined as $>8$ leukocytes in urinary sediment or clinical symptoms of UTI; New York Heart Association (NYHA) heart insufficiency class 3 or 4; uncontrolled diabetes mellitus (glycated hemoglobin $(\mathrm{HbA} 1 \mathrm{C}$ ) $>8.5 \%$ ); triglyceride and/or total cholesterol concentration $>300 \mathrm{mg} / \mathrm{dL}$; chronic therapy with drugs with confirmed nephrotoxicity, excluding sporadic use of non-steroidal anti-inflammatory drugs; use of sympathomimetics; chronic use of sleep 
Table 1. Clinical characteristics of the whole study population and the subgroups of patients with mild and moderate-to-severe OSA

\begin{tabular}{|c|c|c|c|c|}
\hline Variable & All patients & $\begin{array}{l}\text { Patients with } \\
\text { mild OSA }\end{array}$ & $\begin{array}{l}\text { Patients with moderate-to-severe } \\
\text { OSA }\end{array}$ & $\begin{array}{l}\text { p-value (mild vs moderate-to- } \\
\text { severe OSA) }\end{array}$ \\
\hline Number of patients & 86 (100\%) & 45 (52.3\%) & $\begin{array}{l}41(47.7 \%) \text {, including } 13 \text { with mild } \\
\text { OSA and } 28 \text { with severe OSA }\end{array}$ & \\
\hline Age [years] & $44.6 \pm 10.6$ & $41.9 \pm 11.8$ & $47.5 \pm 8.5$ & $p=0.01$ \\
\hline BMI $\left[\mathrm{kg} / \mathrm{m}^{2}\right]$ & $34.5 \pm 6.0$ & $32.5 \pm 5.4$ & $36.7 \pm 6.1$ & $p=0.02$ \\
\hline WHR & $1.05 \pm 0.08$ & $1.02 \pm 0.08$ & $1.08 \pm 0.07$ & $p=0.02$ \\
\hline $\mathrm{eGFR}\left[\mathrm{mL} / \mathrm{min} / 1.73 \mathrm{~m}^{2}\right]$ & $81.2 \pm 13.8$ & $82.3 \pm 14.8$ & $79.9 \pm 13.0$ & $p=0.03$ \\
\hline $\begin{array}{l}\text { Blood pressure [mm Hg] } \\
\text { systolic } \\
\text { diastolic }\end{array}$ & $\begin{array}{l}137.6 \pm 14.9 \\
85.5 \pm 9.6\end{array}$ & $\begin{array}{c}135.8 \pm 12.0 \\
84.9 \pm 9.0\end{array}$ & $\begin{array}{l}139.5 \pm 17.6 \\
86.1 \pm 10.4\end{array}$ & - \\
\hline $\begin{array}{l}\text { Hypertension and/or use } \\
\text { of antihypertensive medication }\end{array}$ & 45 (52.3\%) & $20(44.4 \%)$ & $25(61 \%)$ & - \\
\hline Diabetes mellitus & $11(12.8 \%)$ & $4(8.9 \%)$ & $7(17.1 \%)$ & - \\
\hline Coronary artery disease & $11(12.8 \%)$ & $7(24.1 \%)$ & $4(9.76 \%)$ & - \\
\hline $\begin{array}{l}\text { Number of patients with urine protein } \\
\text { excretion }>0.3 \mathrm{~g} / \mathrm{L}\end{array}$ & $9(10.5 \%)$ & $3(6.7 \%)$ & $6(14.6 \%)$ & - \\
\hline Active smokers & $22(25.6 \%)$ & $10(22.2 \%)$ & $12(29.3 \%)$ & - \\
\hline $\begin{array}{l}\text { Antihypertensive treatment } \\
\text { ACEI } \\
\text { ARB } \\
\text { CCB } \\
\text { BB } \\
\text { a blockers } \\
\text { loop diuretics } \\
\text { thiazide diuretics } \\
\text { AA }\end{array}$ & $\begin{array}{c}40(46.5 \%) \\
26(30.2 \%) \\
9(10.5 \%) \\
13(15.1 \%) \\
16(18.6 \%) \\
5(5.8 \%) \\
17(19.8 \%) \\
2(2.3 \%)\end{array}$ & $\begin{array}{c}17(37.7 \%) \\
10(17.2 \%) \\
3(10.3 \%) \\
3(10.3 \%) \\
7(15.5 \%) \\
1(2.2 \%) \\
5(11.1 \%) \\
1(2.2 \%)\end{array}$ & $\begin{array}{c}23(56.1 \%) \\
16(39.0 \%) \\
6(14.6 \%) \\
10(24.4 \%) \\
9(21.9 \%) \\
4(9.8 \%) \\
12(29.3 \%) \\
1(2.4 \%)\end{array}$ & - \\
\hline Lipid-lowering therapy & $18(20.9 \%)$ & $9(20.0 \%)$ & $9(21.9 \%)$ & - \\
\hline AHI (events per $1 \mathrm{~h}$ of recorded sleep time) & $32.1 \pm 28.5$ & $11.2 \pm 12.3$ & $38.3 \pm 20.7$ & $p=0.000$ \\
\hline
\end{tabular}

OSA - obstructive sleep apnea; BMI - body mass index; WHR - waist-hip ratio; eGFR - estimated glomerular filtration rate; CKD - chronic kidney disease; AHI - apnea-hypopnea index; ACEI - angiotensin-converting enzyme inhibitors; ARB - angiotensin receptor blockers; CCB - calcium channel blockers; $\mathrm{BB}$ - beta-adrenergic receptor blockers; AA - aldosterone receptor antagonists.

medications; alcohol abuse; liver disease; or chronic obstructive and restrictive pulmonary diseases. Subjects with uncontrolled arterial hypertension with systolic blood pressure (SBP) $>160 \mathrm{~mm} \mathrm{Hg}$ and diastolic blood pressure (DBP) $>100 \mathrm{~mm} \mathrm{Hg}$ were also excluded.

The research was conducted in accordance with the Declaration of Helsinki, and the study protocol was approved by the ethics committee at the Medical University of Łódź. All the participants signed informed consent prior to their recruitment.

The subjects' blood pressure was measured with an electronic automatic sphygmomanometer (Omron M1 Plus; Omron Healthcare Co. Ltd., Kyoto, Japan) twice, before and after each diagnostic night. For the blood pressure analysis, the values of the mean arterial pressure (MAP) were calculated with the standard formula: MAP $=[(\mathrm{DBP}$ $+1 / 3 \%(\mathrm{SBP}-\mathrm{DBP})]$. The eGFR was calculated from serum creatinine using the CKD-EPI formula: eGFR $\mathrm{CKD}_{\text {-EPI }}$ $=141 \times \min \left(\mathrm{S}_{\mathrm{cr}} / \mathrm{\kappa}, 1\right)^{\alpha} \times \max \left(\mathrm{S}_{\mathrm{cr}} / \mathrm{\kappa}, 1\right)^{-1.209} \times 0.993^{\mathrm{age}}$.

Forty-one patients diagnosed with moderate-to-severe OSA were qualified for treatment with CPAP (RemStar Plus CPAP Machine; Respironics, Murrysville, USA). They all received 6-8 weeks of nightly CPAP treatment after appropriate training and a titration period. The CPAP therapy was supervised by one of the authors (P.B.). Compliance with the therapy was checked throughout the treatment, and use of the CPAP device for $\geq 4$ h per night was considered acceptable compliance. The remaining 45 patients with mild OSA did not receive CPAP treatment and were managed conservatively. Figure 1 shows the design of the study and a flowchart of the patients; Table 1 presents the participants' clinical characteristics.

The patients were asked to come to our sleep lab in the evening before their polysomnography (PSG) between 7:00 and 9:00 pm to sign the consent form and to receive detailed instructions about the study protocol, including lab sample collections. At that time, baseline blood and urine samples were taken. The $2^{\text {nd }}$ biochemical blood and urine samples were taken in the morning after the diagnostic night, after the completion of all the procedures, at around 7:00-8:00 am. Serum creatinine, total and low-density lipoprotein (LDL) cholesterol, triglycerides, serum high-sensitivity C-reactive protein (hsCRP), and endothelium marker intercellular adhesion molecule-1 (ICAM-1) were measured at baseline in the evening before the diagnostic night and all the measurements were 


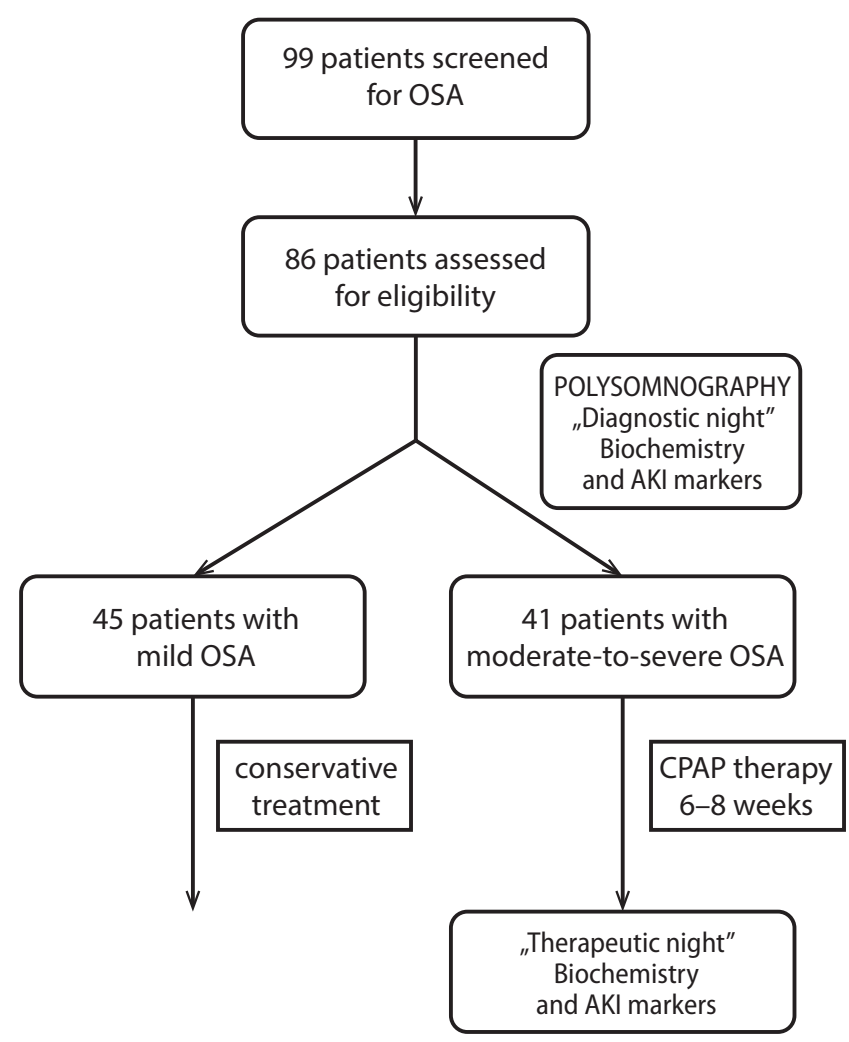

Fig. 1. Flowchart showing the study design

repeated the morning after the diagnostic night. Urine markers including creatinine, cystatin C, NGAL, L-FABP, and KIM-1 were assessed in a sample of urine given before polysomnography protocol was started and in the morning after the polysomnography. The results of the measurements of biomarkers in urine were expressed as ratios per gram of urine creatinine to avoid the differences in the time and volume of urine collection. In the patients with mild-to-moderate OSA qualified for CPAP therapy, the same measurements were repeated in the morning after 6-8 weeks of the therapy. All the patients who were initially qualified completed the study procedures.

Serum and urine samples were stored at $-70^{\circ} \mathrm{C}$ until analysis. All the measurements were performed in duplicate. The intra- and inter-assay coefficients of variation were below $5 \%$ for the biomarkers that were measured. All the laboratory assessments were performed in 1 batch in our university central research lab to minimize analytic variations. Urine cystatin $C$ was measured using an enzyme-linked immunosorbent assay (ELISA) (cystatin C human ELISA kits; BioVendor LLC, Candler, USA). Urinary NGAL was also measured with ELISA kits (Lipocalin-2/ NGAL Human ELISA kits; BioVendor LLC). The KIM-1 and L-FABP were assessed with KIM-1 Human ELISA and L-FABP human ELISA kits (R\&D Systems Clinical Controls, Inc., Minneapolis, USA). Serum hsCRP and ICAM1-1 were measured using hsCRP Human ELISA kits and sICAM-1 Human ELISA kits respectively (both from BioVendor, LLC). Data regarding urine excretion of cystatin C,
NGAL, L-FABP, and KIM-1 were expressed as nanograms per gram of creatinine. Serum and urine creatinine, total cholesterol, HDL- and LDL-cholesterol, triglycerides were measured using standard automated laboratory methods in our university central research lab using an Olympus AU 680 multianalyzer (Olympus Corp., Tokyo, Japan).

The statistical analysis was performed with STATISTICA AXAP software, v. 9.1PL (StatSoft Polska, Kraków, Poland). The results were expressed as mean \pm standard deviation (SD), and absolute changes of the variables as mean (95\% confidence interval (95\% CI)). P-values $<0.05$ were considered significant. The t-test for independent samples was used to test between-group differences. For non-normally distributed variables, the Mann-Whitney rank test was used. For intragroup comparisons, a one-way analysis of variance (ANOVA) was used. Qualitative variables between groups were analyzed using $X^{2}$ test. Spearman's test was used to analyze the correlations between AHI and urine biomarkers of AKI (NGAL, KIM-1, L-FABP, cystatin C) and serum ICAM-1.

\section{Results}

The patients with moderate-to-severe OSA and mild OSA did not differ with respect to SBP and DBP, MAP, proteinuria, mean plasma glucose level, serum hsCRP, fasting total cholesterol, triglycerides and LDL cholesterol concentration, hemoglobin concentration, or incidence of arterial hypertension. As shown in Table 1, significant differences between the groups were found with respect to age, body mass index (BMI), waist-to-hip ratio (WHR), and eGFR. The patients with moderate-to-severe OSA were significantly older than the patients with mild OSA. Body mass, BMI and WHR were significantly higher in the moderateto-severe OSA patients than in the mild OSA group. Baseline eGFR was lower in moderate-to-severe OSA patients compared to mild OSA patients.

As shown in Table 2, urine cystatin $C$ was unchanged after the polysomnography night in the whole study group and in both subgroups. Urine cystatin $\mathrm{C}$ measured in the first morning sample of urine after 6-8 weeks of CPAP therapy decreased significantly.

Urine L-FABP did not change significantly throughout the study, including after 6-8 weeks of CPAP therapy in the moderate-to-severe OSA subgroup (Table 2). Urine NGAL excretion was significantly higher after the diagnostic night in the whole study group $(\mathrm{p}=0.04)$ and in the mild OSA patients ( $\mathrm{p}=0.03$ ) (Table 2). Urine KIM-1 excretion significantly increased after the diagnostic night in patients with mild OSA $(p=0.000)$ and in the whole study group ( $\mathrm{p}=0.001)$, but not in the patients with more severe OSA. No significant changes of urine KIM-1 were observed after 6-8 weeks of CPAP treatment (Table 2). Serum ICAM-1 significantly decreased after the diagnostic night in the mild OSA group $(\mathrm{p}=0.02)$, as well after 


\begin{tabular}{|c|c|c|c|c|c|c|c|c|}
\hline & $\begin{array}{l}\frac{0}{0} \\
\frac{0}{0} \\
\frac{1}{ \pm} \\
\frac{0}{L} \\
\frac{1}{U} \\
4\end{array}$ & 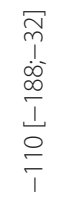 & 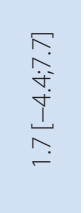 & 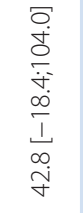 & 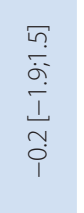 & 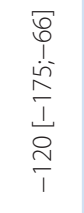 & 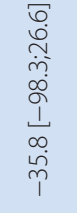 & 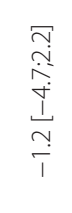 \\
\hline $\begin{array}{l}\mathbb{\sigma} \\
0 \\
\frac{\omega}{\omega}\end{array}$ & 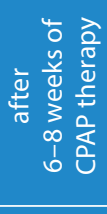 & $\begin{array}{l}\bar{j} \\
\bar{j} \\
+1 \\
o \\
\text { m }\end{array}$ & $\begin{array}{l}m \\
\stackrel{1}{+} \\
+1 \\
m \\
\tilde{=}\end{array}$ & 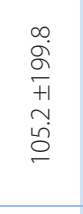 & 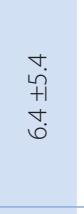 & 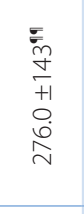 & 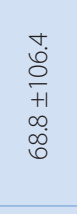 & $\begin{array}{l}\stackrel{\bigcirc}{\stackrel{一}{+}} \\
\underset{+1}{+} \\
\stackrel{+}{\infty}\end{array}$ \\
\hline 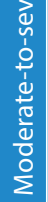 & 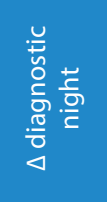 & 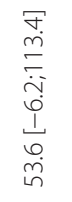 & 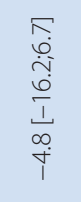 & 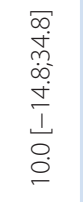 & 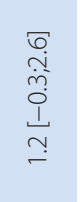 & 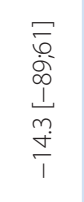 & 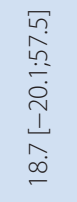 & 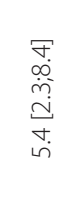 \\
\hline & 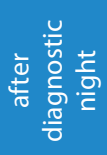 & 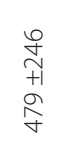 & $\begin{array}{l}n \\
\infty \\
+1 \\
0 \\
\stackrel{1}{0}\end{array}$ & $\begin{array}{l}m \\
\tilde{N} \\
0 \\
+1 \\
\stackrel{1}{0} \\
\tilde{b}\end{array}$ & $\begin{array}{l}0 \\
\dot{L} \\
+1 \\
b \\
\mathscr{b}\end{array}$ & 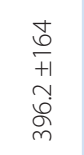 & 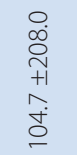 & 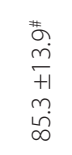 \\
\hline & 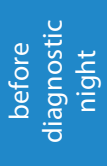 & 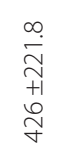 & $\begin{array}{l}m \\
\stackrel{m}{m} \\
m \\
+1 \\
+ \\
\dot{+} \\
\pm\end{array}$ & $\begin{array}{l}\vec{j} \\
\infty \\
\stackrel{1}{+1} \\
+1 \\
\stackrel{N}{i}\end{array}$ & $\begin{array}{l}0 \\
+ \\
+ \\
+1 \\
\text { in } \\
\end{array}$ & 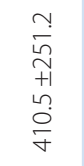 & 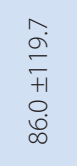 & 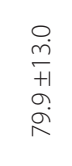 \\
\hline & 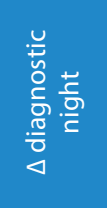 & 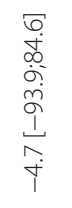 & 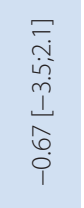 & 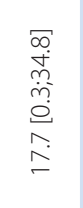 & $\begin{array}{l}\bar{m} \\
\dot{\sigma} \\
\dot{\sigma} \\
\Xi \\
\bar{m}\end{array}$ & $\begin{array}{l}7 \\
0 \\
1 \\
\dot{\sigma} \\
\frac{1}{1} \\
\frac{1}{1} \\
\overline{0} \\
\frac{1}{1}\end{array}$ & 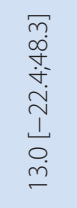 & 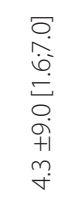 \\
\hline $\begin{array}{l}\frac{\pi}{0} \\
\frac{0}{0} \\
\frac{0}{\Sigma}\end{array}$ & 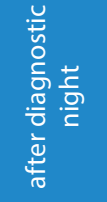 & $\begin{array}{l}\infty \\
\infty \\
\stackrel{+1}{+} \\
\underset{\sim}{+}\end{array}$ & $\begin{array}{l}+ \\
\infty \\
+1 \\
\text { oे } \\
2 \\
\sigma\end{array}$ & 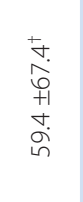 & $\begin{array}{l} \pm \\
\text { in } \\
\text { Ln } \\
+1 \\
0 \\
\text { ํ. }\end{array}$ & 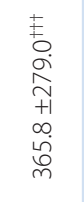 & $\begin{array}{l}\text { va } \\
\infty \\
\frac{\infty}{+1} \\
0 \\
\infty \\
\infty \\
\infty\end{array}$ & 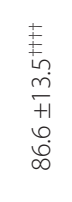 \\
\hline & 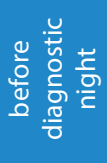 & $\begin{array}{l}\bar{\infty} \\
\stackrel{+}{+1} \\
\dot{v} \\
\stackrel{\gamma}{\sim}\end{array}$ & 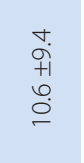 & $\begin{array}{l}\hat{\jmath} \\
\dot{y} \\
+1 \\
\hat{+} \\
\dot{\sigma}\end{array}$ & $\begin{array}{l}0 \\
m \\
+1 \\
\stackrel{+}{0} \\
\dot{m}\end{array}$ & $\begin{array}{l}0 \\
\dot{0} \\
+ \\
+1 \\
\infty \\
0 \\
\dot{0} \\
\dot{q}\end{array}$ & $\begin{array}{l}\hat{0} \\
\frac{0}{+} \\
+1 \\
\stackrel{ }{2}\end{array}$ & 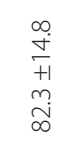 \\
\hline & 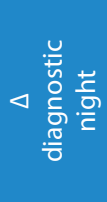 & 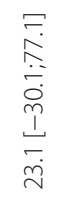 & 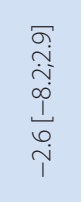 & $\begin{array}{l}\sigma \\
0 \\
\infty \\
\\
\stackrel{0}{0} \\
\dot{0} \\
\dot{+}\end{array}$ & 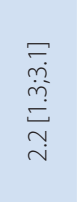 & 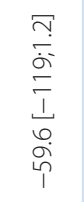 & $\begin{array}{l}m \\
m \\
\dot{J} \\
\sigma \\
\sigma \\
1 \\
\\
\hat{n}\end{array}$ & 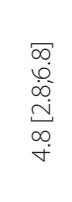 \\
\hline $\bar{\varepsilon}$ & 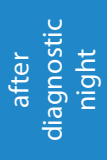 & 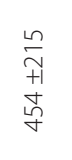 & $\begin{array}{l}m \\
\infty \\
+1 \\
\infty \\
\infty\end{array}$ & 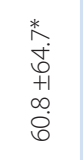 & 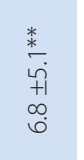 & $\begin{array}{l}\stackrel{\infty}{ \pm} \\
+1 \\
m \\
\infty \\
\infty \\
m\end{array}$ & $\begin{array}{l}\stackrel{n}{n} \\
\sigma \\
\frac{1}{+1} \\
m \\
\end{array}$ & 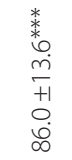 \\
\hline & 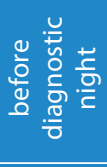 & $\begin{array}{l}\stackrel{N}{\mathscr{T}} \\
+1 \\
\bar{y}\end{array}$ & $\begin{array}{l}\underset{\text { İ }}{+} \\
+1 \\
\stackrel{+}{ \pm}\end{array}$ & $\begin{array}{l}\stackrel{9}{u} \\
\dot{0} \\
+1 \\
\infty \\
o \\
\dot{\sigma}\end{array}$ & $\begin{array}{l}\underset{+}{+} \\
+1 \\
o \\
\forall\end{array}$ & 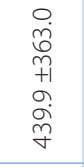 & 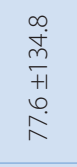 & $\begin{array}{l}\stackrel{\infty}{m} \\
\stackrel{+}{+1} \\
\stackrel{+}{\infty} \\
\infty\end{array}$ \\
\hline & $\begin{array}{l}\frac{0}{0} \\
\frac{0}{\frac{0}{2}} \\
\frac{0}{5}\end{array}$ & 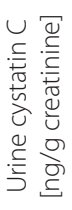 & 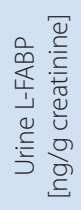 & 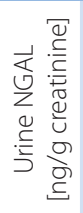 & 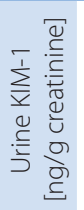 & 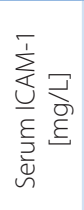 & 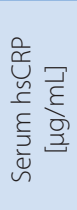 & 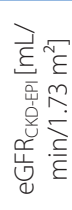 \\
\hline
\end{tabular}

ᄃ

드 உ

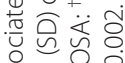

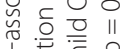

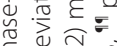
둥 중

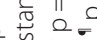
$+1+\frac{1}{4}$ है $\overline{8}$ (II 过 它糔 훙ㅎㅇ

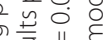
我芒

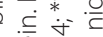

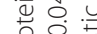
일 主* ${ }^{*} \cdot \frac{\pi}{7}$

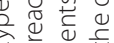

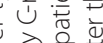
$\sum_{\overline{0}} \overline{\bar{\sigma}}$ के $\overline{2}$ 立.웜

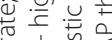
등 원는 递 헝 造离
6-8 weeks of CPAP therapy in the moderate-to-severe OSA group $(\mathrm{p}=0.002)$ (Table 2$)$. Serum hsCRP did not change significantly during the diagnostic night or in the further course of the study (Table 2).

Systolic blood pressure did not change significantly at any time during the study. A significant decrease in DBP was observed after the diagnostic night in both the mild OSA patients $(\mathrm{p}=0.005)$ and the moderate-to-severe OSA patients $(\mathrm{p}=0.005)$. Diastolic blood pressure also decreased significantly after 6-8 weeks of CPAP treatment in the moderateto-severe OSA group. Similar significant changes were seen in MAP (Table 3).

Before the CPAP treatment, there was a significant positive correlation between $\mathrm{AHI}$ and the absolute change in serum ICAM-1 $(\mathrm{R}=0.27, \mathrm{p}=0.009)$ and urine cystatin $C(R=0.27, p=0.009)$ in all the subjects. After the 6-8 weeks CPAP intervention, a significant negative correlation between AHI and the absolute change in urine L-FABP concentration $(\mathrm{R}=-0.35, \mathrm{p}=0.02)$ was revealed.

\section{Discussion}

The results of our study only partially support the concept that OSA may result in subclinical AKI and that the treatment of OSA with CPAP may reduce the extent of kidney damage and decrease blood pressure. The latter finding is not unexpected, since the treatment of OSA with CPAP resulted in a significant decrease in glomerular pressure and hyperfiltration. ${ }^{11}$ Comparable results were presented by Koga et al., who evaluated 27 patients with OSA but without CKD both before and 3 months after CPAP therapy, and found a small but a significant improvement in eGFR. ${ }^{13}$ In our study, there was a small but significant overnight increase in eGFR in the patients with mild OSA, and that finding may also indirectly support the concept of hyperfiltration caused by apneic episodes during sleep. In most of the studies that have been carried out so far, the impairment of kidney function in patients with OSA was assessed only with changes in standard markers such as the eGFR, serum creatinine, urinary albumin excretion, or a sodium thiosulphate and para-aminohippurate (PAH) double clearance test. ${ }^{11,13,14}$ We were able to identify only 1 very recent publication in which a similar protocol to our study was used, showing elevated serum cystatin $\mathrm{C}$ and NGAL in patients with AKI at baseline, and the levels of these markers were positively correlated with OSA severity. ${ }^{15}$ Similar relations were not confirmed in our study, and that also applied to other sensitive AKI markers that we measured, including KIM-1 and L-FABP. The choice of AKI biomarkers 
Table 3. Systolic, diastolic and mean blood pressure in the patients with OSA and in the subgroups of patients with mild and moderate-to-severe OSA

\begin{tabular}{|c|c|c|c|c|c|c|c|c|}
\hline \multirow[b]{2}{*}{ Parameter } & \multicolumn{2}{|c|}{ All OSA } & \multicolumn{2}{|c|}{ Mild OSA } & \multicolumn{4}{|c|}{ Moderate-to-severe OSA } \\
\hline & $\begin{array}{c}\text { before } \\
\text { diagnostic } \\
\text { night }\end{array}$ & $\begin{array}{c}\text { after } \\
\text { diagnostic } \\
\text { night }\end{array}$ & $\begin{array}{c}\text { before } \\
\text { diagnostic } \\
\text { night }\end{array}$ & $\begin{array}{c}\text { after } \\
\text { diagnostic } \\
\text { night }\end{array}$ & $\begin{array}{c}\text { before } \\
\text { diagnostic } \\
\text { night }\end{array}$ & $\begin{array}{c}\text { after } \\
\text { diagnostic } \\
\text { night }\end{array}$ & $\begin{array}{c}\text { before } \\
\text { therapeutic } \\
\text { night }\end{array}$ & $\begin{array}{c}\text { after } \\
\text { therapeutic } \\
\text { night }\end{array}$ \\
\hline $\begin{array}{l}\text { SBP } \\
{[\mathrm{mm} \mathrm{Hg}]}\end{array}$ & $137.6 \pm 14.9$ & $136.5 \pm 14.7$ & $135.8 \pm 12.0$ & $135.2 \pm 12.3$ & $139.5 \pm 17.6$ & $138.1 \pm 17.1$ & $137.8 \pm 14.5$ & $135.8 \pm 13.0$ \\
\hline $\begin{array}{l}\text { DBP } \\
{[\mathrm{mm} \mathrm{Hg}]}\end{array}$ & $85.5 \pm 9.6$ & $82.5 \pm 9.4^{*}$ & $84.9 \pm 9.0$ & $81.8 \pm 8.5^{\text {fキ }}$ & $86.1 \pm 10.4$ & $83.2 \pm 10.4^{\neq \neq}$ & $83.8 \pm 10.6^{\#}$ & $81.2 \pm 9.3^{* *}$ \\
\hline $\begin{array}{l}\text { MAP } \\
{[\mathrm{mm} \mathrm{Hg}]}\end{array}$ & $102.8 \pm 10.6$ & $100.5 \pm 10.5^{\text {fキ }}$ & $101.8 \pm 9.1$ & $99.6 \pm 9.1^{\dagger}$ & $103.9 \pm 12.2$ & $101.5 \pm 12.0^{\dagger}$ & $101.7 \pm 11.4^{\# \#}$ & $100.9 \pm 10.7$ \\
\hline
\end{tabular}

OSA - obstructive sleep apnea; SBP - systolic blood pressure; DBP - diastolic blood pressure; MAP - mean arterial pressure. Significant differences after vs before the diagnostic night: ${ }^{*} p=0.001 ;{ }^{* *} p=0.000 ;{ }^{\dagger} p=0.002 ;{ }^{* \neq} p=0.005$. Significant differences before therapeutic night No. 1 vs before diagnostic night: ${ }^{*} \mathrm{p}=0.003 ;{ }^{\# \#} \mathrm{p}=0.002$

in our research included the 4 most validated biomarkers of AKI. ${ }^{7,8}$ The biomarkers of AKI could be measured both in the urine and the plasma., ${ }^{7,8}$ In our study, in contrast to a study by Chuang et al., ${ }^{15}$ we decided to measure urine markers of AKI, because most data from the literature have confirmed that in contrast to measurements in urine, plasma biomarkers show less specificity for AKI, since they also affected by non-renal factors such as the inflammatory state itself. 7,16

The time course of the changes in each biomarker after AKI is different. ${ }^{7,8,16}$ That important fact was taken into account in our study. We were, however, limited by the timing of the routine diagnostics of OSA and CPAP treatment. Urinary NGAL has been found to increase 15-fold within $2 \mathrm{~h}$ and 25 -fold $4 \mathrm{~h}$ and $6 \mathrm{~h}$ after cardiac surgery; urine L-FABP increased $6 \mathrm{~h}$ after an injury, and urine cystatin $C$ within 6 h; while urine KIM-1 had 90\% sensitivity to detect cardiac surgery-associated AKI when tested $2 \mathrm{~h}$ and $6 \mathrm{~h}$ after surgery. ${ }^{7,17-19}$ However, the complete time course of urinary levels for each urinary biomarker in AKI due to different or multiple renal injuries is unknown. ${ }^{7}$ The samples in our study were collected after $8 \mathrm{~h}$ overnight PSG, during which potential repeated AKIs caused by episodes of apnea were expected to occur.

McIlroy et al. found that urine NGAL best identified AKI in patients with normal baseline renal function. ${ }^{20}$ In that study, among the patients with baseline eGFR $\geq 60 \mathrm{~mL} / \mathrm{min}$, urinary NGAL was higher in those who developed AKI. ${ }^{20}$ Our study population included only patients with $\mathrm{eGFR}_{\mathrm{CKD} \text {-EPI }} \geq 60 \mathrm{~mL} / \mathrm{min}$; thus, the potential effect of OSA on subclinical kidney injury should have been noticeable in assessments of urine NGAL. Urine NGAL increased significantly during the diagnostic night in the whole study population and in the patients with mild OSA. However, in patients with more advanced OSA, no significant changes in urine NGAL were seen during the diagnostic night, and 6-8 weeks of CPAP treatment induced no significant effects on that parameter of kidney injury. We can only speculate that the lack of effect of CPAP on urine NGAL in the patients with more advanced OSA may be due to the fact that more severe disease may be associated with longer and more intensive ischemic "preconditioning" of the kidneys, making them resistant to subtle insults caused by frequent apneic episodes during the night. The results on the relationship between NGAL as a marker of AKI and the severity of OSA have been conflicting since Cheung et al..$^{15}$ reported a relationship between the two, but the authors of another study found that plasma NGAL may not serve as a specific biomarker of OSA in clinical practice, because the kidney injury induced by OSA may only slightly contribute to systemic NGAL secretion; serum NGAL levels appeared to be influenced largely by other factors. ${ }^{21}$ We tried to overcome that limitation by measuring urine NGAL instead of serum NGAL, but we were unable to identify any significant association between AHI and the overnight change in NGAL in our patients.

Urinary cystatin $C$ excretion is another biomarker of AKI, since its excretion increases when the reabsorptive capacity of proximal tubular cells is impaired due to AKI. ${ }^{7}$ There is little information on the association of OSA with serum or urine cystatin $C$ levels. In our study, urine cystatin $\mathrm{C}$ significantly decreased after 6-8 weeks of CPAP treatment, which may suggest that the treatment provides some nephroprotection, but longer observations would be needed to confirm that effect. Most studies to date have investigated serum levels rather than the urine excretion of this biomarker and have been focused on chronic rather than acute effects. Zhang et al. performed a cross-sectional study and showed that serum cystatin $C$ was associated with the severity of OSA in younger men. ${ }^{22}$ In another study by Zhang et al., patients with severe OSA were recruited and treated with CPAP for 3 months, and the authors assessed serum cystatin C, creatinine and eGFR after the CPAP treatment. That study demonstrated that CPAP can decrease cystatin $C$ levels in severe OSA patients and may prevent latent renal impairment. ${ }^{23}$ Additionally, Kato et al. found that severe OSA was independently associated with increased serum cystatin $\mathrm{C}$ concentration. ${ }^{24}$ Chuang et al. ${ }^{15}$ did not find any significant change of serum cystatin $\mathrm{C}$ after 6 months of CPAP treatment. 
Most novel biomarkers of AKI, such as urinary KIM-1 and L-FABP, have not yet been extensively investigated. We were unable to identify any studies in which these novel biomarkers have been investigated in patients with OSA.

Serum ICAM-1 has traditionally been linked to endothelial dysfunction associated with vascular injury, which is associated with OSA. ${ }^{25}$ In our study, we found that 6-8 weeks of CPAP treatment resulted in a significant decrease in serum ICAM-1 concentration in the patients with moderate-to-severe OSA. In 2 previous studies, serum ICAM-1 was higher in patients with $\mathrm{OSA}^{26,27}$ and showed a positive correlation with the severity of OSA and in particular with the intensity of nocturnal hypoxemia episodes. ${ }^{27}$ In a multiple logistic regression analysis, Ursavaş et al. revealed that OSA was associated with high ICAM-1 levels independently of age, sex, BMI, smoking status, and cardiovascular disease. ${ }^{28}$ Ohga et al. measured circulating ICAM-1 levels before and after long-term CPAP therapy in patients with OSA. ${ }^{29}$ They reported that 8 months of CPAP treatment was associated with a significant decrease in serum ICAM-1, and that ICAM-1 was significantly related to oxygen desaturation and nasal CPAP therapy. The influence of CPAP treatment on ICAM-1 levels was also studied by Chin et al. ${ }^{30}$ They confirmed that CPAP therapy significantly decreased serum ICAM-1 after 3 or 4 days, 1 month, or 6 months of treatment. Our results were concordant with the results of Wang et al., who confirmed that CPAP treatment decreased levels of ICAM-1 in patients with moderate and severe OSA. ${ }^{31}$

Although our study was not designed to analyze the relationship between OSA and systemic inflammation, we measured serum CRP levels but did not find any significant changes in this parameter after the diagnostic night in either group, or after CPAP therapy in the patients with moderate-to-advanced OSA. This result contrasts with most recent findings. The topic has been intensively studied, and a recent meta-analysis reported that patients with OSA develop a systemic inflammation. ${ }^{32}$

We also demonstrated a significant decrease in DBP and MAP after the diagnostic night in both of the study groups, while CPAP treatment favorably influenced only DBP. The data from the literature is ambiguous. Among the patients with OSA and resistant hypertension in the HIPARCO randomized clinical trial, CPAP treatment for 12 weeks resulted in a decrease in 24-hour MAP and DBP, and an improvement in the nocturnal blood pressure pattern. ${ }^{33}$ Likewise, Durán-Cantolla et al. reported that CPAP produced a small but statistically significant reduction in blood pressure in patients with systemic hypertension and OSA. ${ }^{34}$ On the contrary, Muxfeldt et al. found that CPAP treatment had no significant effect on clinic or ambulatory blood pressure in patients with resistant hypertension and moderate-to-severe OSA. ${ }^{35}$

Our study has several strengths. This is the first study that has comprehensively analyzed most novel specific urine
AKI biomarkers, including NGAL, KIM-1, L-FABP, and cystatin $\mathrm{C}$, in patients with recurrent episodes of hypoxia and hypercapnia during sleep apneic episodes. Recruiting males for the study meant we assembled a homogenous population and avoided significant sex-related differences in several biomarkers, including urinary NGAL, of which women have higher levels. ${ }^{36} \mathrm{We}$ also avoided age-related differences in urinary NGAL levels and the effect of age-related kidney function decline, since we enrolled only males aged 40-59. We also carefully excluded patients with leukocyturia, systemic inflammation, glucocorticoid therapy, advanced CKD, and proteinuria $>1.0 \mathrm{~g} / 24 \mathrm{~h} / 1.73 \mathrm{~m}^{2}$ body surface, which may have influenced the results of urinary NGAL and cystatin C.

Our study has also several limitations. The issue of the time lag between renal injury and urine sampling may have had a significant impact on the results, since urinary NGAL levels vary greatly depending on their proximity in time to ischemic injury. In studies where NGAL was measured after cardiac bypass surgery, NGAL was elevated for at least $24 \mathrm{~h}$ after injury, but appeared to peak at $\sim 6 \mathrm{~h}$ after an insult. Therefore, if the injury occurred acutely or a long time after OSA, an increase in urinary NGAL may have been missed. A relatively short duration of CPAP therapy may also be a limitation of the study. Even with the biomarker-aided improvement in risk stratification, we are unable to assess the potential implications of CPAP for clinical management without a prospective randomized trial.

In summary, we found that OSA may cause subclinical AKI, mainly in patients with mild OSA. The CPAP therapy may reduce the extent of the injury, but prospective randomized trials are required to confirm the potential implications of our results for the management of sleep apnea in patients with renal function impairment.

\section{ORCID iDs}

Michał Nowicki (D) https://orcid.org/0000-0002-0823-5440 Anna Zawiasa-Bryszewska (B) https://orcid.org/0000-0002-7964-4268 Małgorzata Taczykowska (D) https://orcid.org/0000-0002-1066-4412 Piotr Białasiewicz (D) https://orcid.org/0000-0002-8338-1747 Dariusz Nowak (1) https://orcid.org/0000-0001-9273-3970

\section{References}

1. Gonzaga C, Bertolami A, Bertolami M, Amodeo C, Calhoun D. Obstructive sleep apnea, hypertension and cardiovascular diseases. J Hum Hypertens. 2015;29(12):705-712.

2. Foster GE, Poulin MJ, Hanly PJ. Intermittent hypoxia and vascular function: Implications for obstructive sleep apnoea. Exp Physiol. 2007; 92(1):51-65.

3. Domenech P, Perez T, Saldarini A. Uad P, Musso CG. Kidney-lung pathophysiological crosstalk: Its characteristics and importance. Int Urol Nephrol. 2017;49(7):1211-1215.

4. Dou L, Lan H, Reynolds DJ, et al. Association between obstructive sleep apnea and acute kidney injury in critically ill patients: A propensity-matched study. Nephron. 2017;135(2):137-146.

5. Tsioufis C, Thomopoulos C, Dimitriadis K, et al. Association of obstructive sleep apnea with urinary albumin excretion in essential hypertension: A cross-sectional study. Am J Kidney Dis. 2008;52(2):285-293.

6. Agrawal V, Vanhecke TE, Rai B, Franklin BA, Sangal RB, McCullough PA. Albuminuria and renal function in obese adults evaluated for obstructive sleep apnea. Nephron Clin Pract. 2009;113(3):c140-c147. 
7. Kashani K, Cheungpasitporn W, Ronco C. Biomarkers of acute kidney injury: The pathway from discovery to clinical adoption. Clin Chem Lab Med. 2017;55(8):1074-1089.

8. Vanmassenhove J, Vanholder R, Nagler E, Van Biesen W. Urinary and serum biomarkers for the diagnosis of acute kidney injury: An indepth review of the literature. Nephrol Dial Transplant. 2013;28(2): 254-273.

9. Lattimore JL, Wilcox I, Skilton M, Langenfeld M, Celermajer DS. Treatment of obstructive sleep apnoea leads to improved microvascular endothelial function in the systemic circulation. Thorax. 2006;61(6): 491-495.

10. Pépin JL, Tamisier R, Baguet JP, Lévy P. Arterial health is related to obstructive sleep apnea severity and improves with CPAP treatment. Sleep Med Rev. 2013;17(1):3-5.

11. Kinebuchi S, Kazama JJ, Satoh M, et al. Short-term use of continuous positive airway pressure ameliorates glomerular hyperfiltration in patients with obstructive sleep apnoea syndrome. Clin Sci (Lond). 2004;107(3):317-322.

12. Berry RB, Budhiraja R, Gottlieb DJ, et al; American Academy of Sleep Medicine. Rules for scoring respiratory events in sleep: Update of the 2007 AASM Manual for the Scoring of Sleep and Associated Events. Deliberations of the Sleep Apnea Definitions Task Force of the American Academy of Sleep Medicine. J Clin Sleep Med. 2012;8(5):597-619.

13. Koga S, Ikeda S, Yasunaga T, Nakata T, Maemura K. Effects of nasal continuous positive airway pressure on the glomerular filtration rate in patients with obstructive sleep apnea syndrome. Intern Med. 2013;52(3):345-349.

14. Faulx MD, Storfer-Isser A, Kirchner HL, Jenny NS, Tracy RP, Redline S. Obstructive sleep apnea is associated with increased urinary albumin excretion. Sleep. 2007;30(7):923-929.

15. Chuang LP, Lin SW, Lee LA, et al. Elevated serum markers of acute kidney injury in patients with obstructive sleep apnea. J Clin Sleep Med. 2019;15(2):207-213.

16. Soto K, Coelho S, Rodrigues B, et al. Cystatin C as a marker of acute kidney injury in the emergency department. Clin J Am Soc Nephrol. 2010;5(10):1745-1754.

17. Bennett $M$, Dent $C L, M a$, et al. Urine NGAL predicts severity of acute kidney injury after cardiac surgery: A prospective study. Clin J Am Soc Nephrol. 2008;3(3):665-673.

18. Huang Y, Don-Wauchope AC. The clinical utility of kidney injury molecule 1 in the prediction, diagnosis and prognosis of acute kidney injury: A systematic review. Inflamm Allergy Drug Targets. 2011;10(4): 260-271.

19. Shao X, Tian L, Xu W, et al. Diagnostic value of urinary kidney injury molecule 1 for acute kidney injury: A meta-analysis. PLoS One. 2014;9(1):e84131.

20. Mcllroy DR, Wagener G, Lee HT. Neutrophil gelatinase-associated lipocalin and acute kidney injury after cardiac surgery: The effect of baseline renal function on diagnostic performance. Clin J Am Soc Nephrol. 2010;5(2):211-219.
21. Murase K, Mori K, Yoshimura C, et al. Association between plasma neutrophil gelatinase associated lipocalin level and obstructive sleep apnea or nocturnal intermittent hypoxia. PLoS One. 2013;8(1):e54184.

22. Zhang XB, Lin QC, Deng CS, Chen GP, Cai ZM, Chen H. Elevated serum cystatin $C$ in severe OSA younger men without complications. Sleep Breath. 2013;17(1):235-241.

23. Zhang XB, Jiang XT, Lin QC, Chen X, Zeng HQ. Effect of continuous positive airway pressure on serum cystatin C among obstructive sleep apnea syndrome patients. Int Urol Nephrol. 2014;46(10):1997-2002.

24. Kato K, Takata Y, Usui Y, et al. Severe obstructive sleep apnea increases cystatin C in clinically latent renal dysfunction. Respir Med. 2011; 105(4):643-649.

25. Ohga E, Nagase T, Tomita $T$, et al. Increased levels of circulating ICAM-1, VCAM-1 and L-selectin in obstructive sleep apnea syndrome. J Appl Physiol (1985). 1999;87(1):10-14.

26. El-Solh AA, Mador MJ, Sikka P, Dhillon RS, Amsterdam D, Grant BJ. Adhesion molecules in patients with coronary artery disease and moderate-to-severe obstructive sleep apnea. Chest. 2002;121(5):1541-1547.

27. Zamarron-Sanz C, Ricoy-Galbaldon J, Gude-Sampedro F, RiveiroRiveiro A. Plasma levels of vascular endothelial markers in obstructive sleep apnea. Arch Med Res. 2006;37(4):552-555.

28. Ursavaş A, Karadağ M, Rodoplu E, Yilmaztepe A, Oral HB, Gözü RO. Circulating ICAM-1 and VCAM-1 levels in patients with obstructive sleep apnea syndrome. Respiration. 2007;74(5):525-532.

29. Ohga E, Tomita T, Wada H, Yamamoto H, Nagase T, Ouchi Y. Effects of obstructive sleep apnea on circulating ICAM-1, IL-8 and MCP-1. J Appl Physiol (1985). 2003;94(1):179-184.

30. Chin K, Nakamura T, Shimizu K, et al. Effects of nasal continuous positive airway pressure on soluble cell adhesion molecules in patients with obstructive sleep apnea syndrome. Am J Med. 2000;109(7):562-567.

31. Wang YN, Yang Y, Luo YQ, Chen LL. Effects of nasal continuous positive airway pressure short-term treatment on C-reactive protein and intercellular adhesion molecule-1 in patients with obstructive sleep apnea-hypopnea syndrome [in Chinese]. Zhong Nan Da Xue Xue Bao YiXue Ban. 2005;30:666-669.

32. Li K, Wei $P$, Qin $Y$, Wei Y. Is C-reactive protein a marker of obstructive sleep apnea? A meta-analysis. Medicine (Baltimore). 2017;96(19):e6850.

33. Martínez-García MA, Capote F, Campos-Rodríguez F, et al. Effect of CPAP on blood pressure in patients with obstructive sleep apnea and resistant hypertension: The HIPARCO randomized clinical trial. JAMA. 2013;310(22):2407-2415.

34. Durán-Cantolla J, Aizpuru F, Montserrat JM, et al. Continuous positive airway pressure as treatment for systemic hypertension in people with obstructive sleep apnoea: Randomised controlled trial. BMJ. 2010;341:c5991.

35. Muxfeldt ES, Margallo V, Costa LM, et al. Effects of continuous positive airway pressure treatment on clinic and ambulatory blood pressures in patients with obstructive sleep apnea and resistant hypertension: A randomized controlled trial. Hypertension. 2015;65(4):736-742.

36. Cullen MR, Murray PT, Fitzgibbon MC. Establishment of a reference interval for urinary neutrophil gelatinase-associated lipocalin. Ann Clin Biochem. 2012;49(Pt 2):190-193. 